\title{
Impact of Palivizumab on RSV Hospitalizations for Children with Hemodynamically Significant Congenital Heart Disease
}

\author{
Ruey-Kang R. Chang $\cdot$ Alex Y. Chen
}

Received: 18 May 2009/Accepted: 22 October 2009/Published online: 14 November 2009

(c) The Author(s) 2009. This article is published with open access at Springerlink.com

\begin{abstract}
The objective of this study was to evaluate the impact of palivizumab prophylaxis on respiratory syncytial virus (RSV) hospitalizations among children with hemodynamically significant congenital heart disease (CHD). In 2003, the American Academy of Pediatrics (AAP) revised the bronchiolitis policy statement and recommended the use of palivizumab in children $<24$ months old with hemodynamically significant CHD (HS-CHD). California statewide hospital discharge data from years 2000-2002 (pre-AAP policy revision) were compared to those from years 20042006 (post-AAP policy revision). Hospitalizations due to RSV bronchiolitis for children $<2$ years of age were identified by IDC-9 CM codes 4661.1, 480.1, and 079.6 as the Principal Diagnosis. Children with CHD and children with HS-CHD were identified by the codiagnoses. The overall RSV hospitalization rate was 71 per 10,000 children $<2$ years of age. Of all RSV hospitalizations, $3.0 \%$ were among children with CHD, and $0.50 \%$ among children with HS-CHD. HS-CHD patients accounted for $0.56 \%$ of RSV hospitalizations in 2000-2002, compared to $0.46 \%$ RSV hospitalizations in 2004-2006. That represents a 19\% reduction in RSV hospitalizations among HS-CHD patients after 2003. The $19 \%$ decrease in RSV hospitalizations equates to seven fewer hospitalizations (76 hospital days) per year among HS-CHD patients. We conclude that, since the recommendation of palivizumab for children with
\end{abstract}

R.-K. R. Chang ( $\square)$

Division of Cardiology, Department of Pediatrics,

Harbor-UCLA Medical Center, 1000 West Carson Street,

Box 491, Torrance, CA 90509, USA

e-mail: rkchang@ucla.edu

A. Y. Chen

Childrens' Hospital Los Angeles, Department of Pediatrics,

University of Southern California, Los Angeles, CA, USA
HS-CHD in 2003, the impact on RSV hospitalizations in California among HS-CHD patients has been limited. Considering the high cost of palivizumab administration, the cost-benefit of RSV prophylaxis with palivizumab warrants further investigation.

Keywords RSV prophylaxis · Palivizumab · Congenital heart disease

Respiratory syncytial virus (RSV) bronchiolitis is a major cause of hospitalizations for infants and children $<2$ years of age [13]. RSV immune globulin was the first licensed product for prevention of severe RSV infection [16]. RSV immune globulin, which is no longer in production, was not indicated for infants with cyanotic congenital heart disease (CHD) because of worse outcomes in infants with cyanotic CHD as shown in a preapproval clinical trial [14]. Palivizumab (Synagis; Med-Immune, Gaithersburg, MD) is an anti-RSV, humanized murine, monoclonal antibody that aims to provide passive immunity for the recipient [12]. Palivizumab is administered by monthly injections during the RSV season to patients at risk for severe RSV infection [1]. When palivizumab was first studied in children with prematurity, it was shown to reduce RSV-associated hospitalizations by $55 \%$ [15].

The results of a multicenter, randomized, double-blind, placebo-controlled trial of palivizumab in 1287 children with hemodynamically significant CHD (HS-CHD), funded by MedImmune, Inc., were published in 2003 [6]. This study showed a $45 \%$ reduction in RSV hospitalizations $(p=0.003)$ for children given prophylaxis with palivizumab compared with placebo [6]. Two months following the publication of this study, the American Academy of Pediatrics (AAP) revised their policy statement to 
recommend RSV prophylaxis with monthly injections of palivizumab for children age 24 months or younger who have HS cyanotic or acyanotic CHD [2].

Several studies have evaluated the cost-effectiveness of RSV prophylaxis using palivizumab [5, 9, 11]. The estimates of palivizumab effect on RSV-related hospitalizations used in these cost-effectiveness studies were taken from the results of clinical trials under controlled research settings. However, it is unclear whether the results from clinical trials can be achieved in the "real world." Decision makers in health and medical care are increasingly interested in using reliable scientific evidence to support clinical and health policy choices; however, much of the currently available scientific evidence may not be applicable in realworld settings. Practical scientific evidence is essential in improving care and promoting efficient use of finite resources. There is a growing trend toward emphasizing meaningful and practical clinical studies that can help answer real questions faced by decision makers [3].

The objective of this study is to evaluate the actual impact of palivizumab in reducing RSV-related hospitalizations among children with HS-CHD using populationlevel data from California. Specifically, the rate of RSVrelated hospitalizations in the 3 years before the release of the revised AAP statement in 2003 and the rate 3 years after its release were compared.

\section{Methods}

\section{Hospital Discharge Database}

This study used the California statewide, abstracted hospital discharge data from the California Office of Statewide Health Planning and Development (OSHPD). The OSHPD database includes discharges from all nonfederal acute-care hospitals in California. In this study, the OSHPD data on hospital discharges from years 2000-2002 and 2004-2006 were used to assess the changes in RSV hospitalizations 3 years prior to and 3 years after the release of the revised AAP policy statement in 2003. The OSHPD data contain International Classification of Diseases, Ninth Revision, Clinical Modification (ICD-9-CM), discharge diagnosis and procedure codes assigned by California hospitals to each individual discharge during the year. Fields are provided for up to 24 diagnoses and 20 procedures. Although the clinical information is complete in the public release version of the data, some demographic information was masked to protect the privacy of patients.

Of the 6 years included in this study, the first 3 years (2000-2002) were defined as the Prepalivizumab Era, and the last 3 years (2004-2006) were defined as the Palivizumab Era. Six California hospitals were among 76 centers which participated in the multicenter clinical trial of patients with HS-CHD [6]. These hospitals recruited HS-CHD patients for the palivizumab clinical trial between 1998 and 2002. RSV hospitalizations from these six hospitals were identified for sensitivity analysis.

\section{Total RSV Hospitalizations}

Children $<2$ years of age with Principal Diagnosis in the database indicating RSV-related illness were selected for analysis. RSV-related illnesses included RSV bronchiolitis (ICD9-CM code 466.11), RSV pneumonia (480.1), and other RSV (079.6). Severe bronchiolitis cases that required endotracheal intubation (96.04) or continuous mechanical ventilation (code 96.72 or 96.71) were identified using the ICD-9-CM procedure codes.

Overall, $15.3 \%$ of patients' ages were missing or collapsed (categorized as 1-5 years) in our analytic sample from OSHPD: we excluded these observations from the initial analysis but included them in sensitivity analysis. The percentage of cases with missing or collapsed age was $14.3 \%$ in $2000-2002$ and $16.0 \%$ in 2004-2006.

Vital statistics data of California were used to identify the population $<2$ years of age in California for each of the years 2000-2006. The total numbers of RSV-related hospitalizations for each year between 2000 and 2006 were calculated. The rate of RSV hospitalization was calculated as the number of hospitalizations per 10,000 children $<2$ years of age.

\section{Patients with Hemodynamically Significant CHD}

Among the RSV hospitalizations selected, we identify patients with CHD by the 24 codiagnosis fields of each hospitalization for ICD codes 745-747. The 2003 AAP policy statement recommends the use of palivizumab for CHD patients who have HS-CHD, including those who (1) are receiving medication to control congestive heart failure, (2) have moderate to severe pulmonary hypertension, and (3) have cyanotic heart disease. Therefore, we identified a subgroup of patients with HS-CHD among the selected CHD patients with heart failure (ICD9 code 428), pulmonary hypertension (416.0 and 416.8), or cyanotic heart diseases. We designated this subgroup the HS-CHD group, which corresponds to patients with HS-CHD as the target population for palivizumab use per the revised AAP policy statement.

Assessing the Impact of Palivizumab on RSV Hospitalizations

Among all RSV hospitalizations, the number of patients with HS-CHD was identified. Because the severity of RSV season 
varies from year to year, the number of RSV hospitalizations fluctuates from year to year. Therefore, simply comparing the number of HS-CHD patients hospitalized for RSV in 2000-2002 versus 2004-2006 would be prone to biases.

To estimate the impact of 2003 guidelines on RSV hospitalizations among HS-CHD patients, HS-CHD patients were calculated as a percentage of all RSV hospitalizations in the Prepalivizumab Era (2000-2002) and in the Palivizumab Era (2004-2006). This minimized the effect of year-to-year variations in the severity of RSV. For our analysis, we made the assumption that if there had been no revision in AAP bronchiolitis policy in 2003, the proportion of HS-CHD patients among all RSV hospitalizations would be the same for the Palivizumab Era (20042006) as for the Prepalivizumab Era (2000-2002). Thus, we calculated the percentage reduction in RSV hospitalizations among HS-CHD patients as the change in the proportion of HS-CHD among all RSV hospitalizations between 2000 2002 and 2004-2006. The formula is as follows:

$\{[(\mathrm{Nc} 1 / \mathrm{Nt} 1)-(\mathrm{Nc} 2 / \mathrm{Nt} 2)] /(\mathrm{Nc} 1 / \mathrm{Nt} 1)\} \times 100 \%$

where $\mathrm{Nc1}$ is the number of HS-CHD patients hospitalized for RSV in 2000-2002; Nc2, the number of HS-CHD patients hospitalized for RSV in 2004-2006; Nt1, the number of total RSV hospitalizations in 2000-2002; and $\mathrm{Nt} 2$, the number of total RSV hospitalizations in 2004-2006.

\section{Estimating the Cost of RSV Hospitalization}

The average hospital charge among HS-CHD patients per RSV hospitalization was calculated. Because hospital charges are generally higher than the actual cost, a cost-to-charge ratio of 0.58 (derived from a study on influenza hospitalization at a children's hospital in 2002) [7] was used to convert the hospital charges to the costs of hospitalization.

\section{Results}

There were a total of 53,207 RSV-related hospitalizations among children $<2$ years of age in California from 2000 to 2006. The number of hospitalizations fluctuated over the years, ranging from 6336 in 2006 to 8712 in 2002 . When calculated as the rate per 10,000 children $<2$ years of age,

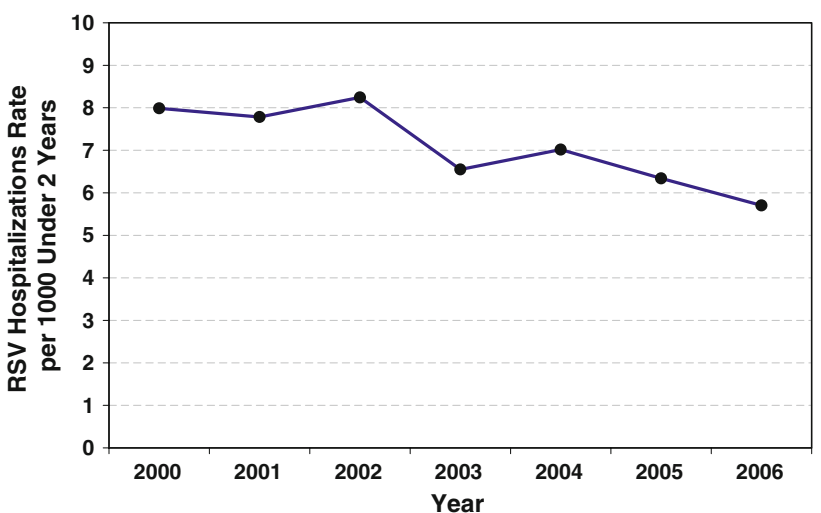

Fig. 1 Annual rate of RSV-related hospitalizations per 1000 infants $<2$ years of age in California, 2000-2006

the overall RSV hospitalization rate was 71 per 10,000 children $<2$ years of age, ranging from 57 per 10,000 in 2006 to 83 per 10,000 in 2002 . The RSV hospitalization rate decreased from 2000 to 2006 (Fig. 1) $(R=0.89$, $p<0.01$ ). Overall, $3.0 \%$ of all RSV hospitalizations were patients with $\mathrm{CHD}$, and $0.5 \%$ of all RSV hospitalizations were patients with HS-CHD.

Table 1 summarizes the RSV hospitalizations among all patients $<2$ years, patients with $\mathrm{CHD}$, and patients with HS-CHD. The length of stay was significantly longer for HS-CHD patients. No trend of changes was noted in the mean or median length of stay over time (Fig. 2). Among the RSV hospitalizations, $2.5 \%$ required intubation or use of a ventilator. The percentage of HS-CHD hospitalizations requiring intubation/ventilator was not significantly different in 2000 $2002(17.9 \%)$ versus 2004-2006 (18.0\%; $p=0.87)$.

There were 36 deaths among the RSV hospitalizations in 2000-2006. The in-hospital mortality rate was $0.07 \%$, or 1 death per 1478 hospitalizations. The in-hospital mortality rate decreased from $0.09 \%$ in $2000-2002$ to $0.06 \%$ in 2004-2006. There were five deaths among HS-CHD patients in California, of which four occurred prior to 2003 and one after 2003.

\section{Impact of Palivizumab Prophylaxis}

We calculated that $0.56 \%$ of all RSV hospitalizations in 2000-2002 were HS-CHD patients, whereas $0.46 \%$ of all RSV hospitalizations in 2004-2006 were HS-CHD

Table 1 Characteristics and outcomes of respiratory syncytial virus (RSV) hospitalizations

\begin{tabular}{lcccc}
\hline RSV hospitalizations & Total $N$ & Length of stay & Intubation $(\%)$ & Mortality \\
\hline All & 53,207 & $3.6 \pm 3.9(3$ days $)$ & 2.6 & $0.07 \%(n=36)$ \\
Among CHD patients & $1,596(3.0 \%)$ & $7.1 \pm 10.3(5$ days $)$ & 9.7 & $0.63 \%(n=10)$ \\
Among HS-CHD patients & $266(0.5 \%)$ & $10.3 \pm 18.0(6$ days $)$ & 18.0 & $1.90 \%(n=5)$
\end{tabular}

CHD congenital heart disease; $H S-C H D$ hemodynamically significant $\mathrm{CHD}$ 


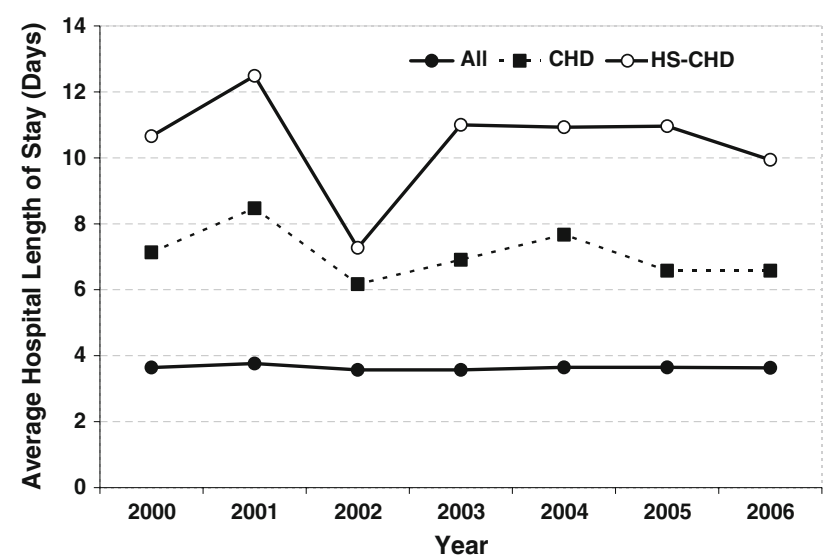

Fig. 2 Average length of stay (days) for all patients $<2$ years of age (filled circles), patients with congenital heart disease (CHD; filled squares), and patients with cyanotic CHD or heart failure (HS-CHD; open circles), 2000-2006

patients. If there had been no policy revision in 2003 to expand palivizumab use, the proportion of RSV hospitalizations with HS-CHD in 2004-2006 would have been $0.56 \%$ (the same as the proportion in 2000-2002). Therefore, there would have been a total of 118 hospitalizations of HS-CHD patients in 2004-2006 (0.56\% of 20,881 total RSV hospitalizations in California in 2004-2006). In 2004-2006, there were 96 observed RSV hospitalizations of HS-CHD patients. Subtracting 96 from 118, we derive 22 fewer hospitalizations for RSV in the Palivizumab Era (or an average of 7 fewer hospitalizations per year) as a result of the AAP policy expansion of palivizumab prophylaxis for HS-CHD patients. The reduction of $22 \mathrm{RSV}$ hospitalizations for HS-CHD patients in 2004-2006 was equivalent to a total of 227 hospital days, that is, a decrease of 76 hospital days per year.

\section{Sensitivity Analysis}

In a sensitivity analysis, the $15.3 \%$ hospitalizations with incomplete age information were included in the analysis, assuming that all of these hospitalizations were among patients $<2$ years of age. The overall hospitalization rate showed a trend of decrease similar to that shown in Fig. 1: from 9.85 hospitalizations per 1000 in 2002 to 6.95 hospitalizations in 2006. In calculating the number of RSV hospitalizations among HS-CHD patients, the reduction in RSV hospitalizations was 21 from the Prepalivizumab Era (2000-2002) to the Palivizumab Era (2004-2006), or an average of 7 fewer hospitalizations per year since 2003 .

Six hospitals in California participated in the palivizumab clinical trial and recruited HS-CHD patients for palivizumab use in the Prepalivizumab Era [6]. The RSV hospitalizations in these hospitals in 2000-2006 accounted for $9.9 \%$ of all RSV hospitalizations. Of HS-CHD patients hospitalized for RSV, $32.2 \%$ were admitted to these six hospitals in the Prepalivizumab Era, which was not significantly different from the $33.3 \%$ in the Palivizumab Era $(p=0.83)$. When these six hospitals were excluded from analysis, it was calculated that the total reduction in HSCHD hospitalizations was 20 hospitalizations from the Prepalivizumab Era to the Palivizumab Era.

\section{Cost of RSV Hospitalization}

The average hospital charge among HS-CHD patients was $\$ 70,365$, and that per RSV hospitalization was $\$ 40,812$. Therefore, the total cost saved in the Palivizumab Era was $\$ 897,864$, from 22 hospitalizations avoided, or a savings of $\$ 299,288$ in California per year.

\section{Discussion}

In the pivotal clinical trial that led to FDA approval of palivizumab use in HS-CHD patients and the AAP policy revision statement in 2003, a reduction in hospitalization rate of $45 \%$ in the palivizumab group, compared with the placebo group, was reported [6]. In the current study, using population-level hospitalization data from California, we found that the reduction in RSV hospitalization rate for HSCHD patients was $<20 \%$, or an equivalent of seven fewer RSV hospitalizations per year for HS-CHD patients.

The overall incidence of RSV hospitalization decreased during the study period 2000-2006 (Fig. 1). The steep decline in RSV incidence after 2003, which temporally correlated with the release of the AAP statement, suggests an effect of palivizumab prophylaxis on overall RSV hospitalizations. Another possible explanation for the decrease in overall RSV hospitalizations during the study period is a change in clinical practice, that those not-sosick patients were not as likely hospitalized in the later era. We were not able to determine the cause of the decline in RSV hospitalizations using our data.

We found that the effect of palivizumab prophylaxis on HS-CHD patients was relatively limited. Why did palivizumab reduce hospitalizations by $45 \%$ in the clinical trial but by only $19 \%$ in the actual hospitalization data from California? A likely explanation for this discrepancy is patient compliance. The administration of palivizumab requires monthly intramuscular injections during the RSV season, typically 5 or 6 months per year. In clinical trials with a well-defined, supported, and controlled research environment, the compliance of health-care providers and patients is "artificially" elevated. In reality, ensuring compliance with a monthly injection regimen is difficult.

While the reduction in hospitalizations of $45 \%$ in a clinical trial seemed impressive and was statistically 
significant, the actual reduction in the number of hospitalizations among 1287 study subjects $(N=639$ in the palivizumab group) was 29 hospitalizations over four RSV seasons, which is a reduction of approximately 7 hospitalizations per year. Considering the high cost of palivizumab use, the additional cost of administering the injections, and the burden on the patients and families, the impact of palivizumab on RSV hospitalizations of HSCHD patients is very limited from a societal point of view.

The question of whether palivizumab use in high-risk infants is cost-effective has been a topic of discussion among some investigators. The first study on the costeffectiveness of palivizumab use among preterm infants was reported by Joffe et al. in 1999 [8]. Joffe et al. used data from the Northern California Kaiser Permanente Medical Care Plan and found that palivizumab prophylaxis would be cost-effective in only one of eight subgroups studied (the subgroup of infants born at 23-32 weeks of gestation, with $>28$ days' oxygen requirement, and neonatal intensive care unit discharge between September and November) [8]. In a study of the North Carolina Medicaid Program, Wegner et al. reported that palivizumab use in infants 32 to 35 weeks of gestation did not provide a cost savings [17]. In fact, the cost per person in the palivizumab prophylaxis group was more than 10 times the per-person cost in the group that did not receive palivizumab prophylaxis ( $\$ 5434$ vs. $\$ 505 ; p<0.0001$ ) [17].

Using a decision-analysis model and a hypothetical cohort of 10,000 CHD patients, Yount and Mahle found that the cost to prevent 1 day of hospitalization is three times the cost of 1 day in the hospital [18]. Of note, one may argue that Yount and Mahle have overestimated the effectiveness of palivizumab in actual practice settings by using the estimates from the MedImmune trial [6], which reported a $45 \%$ reduction in hospitalizations. Using a historical cohort of 527 infants with CHD born in Norway from 1987 to 2004, Meberg and Bruu calculated that the expense of palivizuamab prophylaxis could be as high as 31 times that of hospital treatment [10].

Since the release of the AAP policy statement in 2003 [2], the use of palivizumab for RSV prophylaxis among children with CHD has increased significantly. In the Palivizumab Outcomes Registry (a voluntary report system that does not include all CHD patients receiving palivizumab), the number of subjects with CHD enrolled in the registry increased more than six times from 2000-2001 ( $N=102)$ to 2003-2004 $(N=688)$ [4]. While data after 2004 are not available from the registry, it is likely that the increase in CHD patients continued. The insurance companies and patients will bear the immediate burdens of high palivizumab cost and the unfavorable cost-to-savings ratio. The ultimate burden is passed on to our health-care system. We believe that our findings provide additional population- level information that can be helpful to health-care providers, decision makers, and policymakers in considering the cost and benefit of palivizumab use in children with HS-CHD. Further studies focusing on cost-effectiveness and cost-benefit analyses of palivizumab prophylaxis are needed to guide practice recommendations.

Our study has several limitations. First, we conducted an observational study of a "natural experiment" rather than a prospective experimental study. Observational studies are prone to historical biases and other unmeasured confounders. To truly measure the impact of RSV prophylaxis on children with HS-CHD, the ideal approach would be a population-level experimental design in the real practice settings. However, such a study would be enormously expensive to conduct. Second, our study used an administrative database on hospital discharge. Hospital discharge data are prone to incomplete information and miscoding. The RSV hospitalization rate for children $<2$ years of age in this study was comparable to that in other populationbased studies. Although $15 \%$ of the data were missing information on age, a sensitivity analysis was conducted demonstrating similar results and conclusions. Third, six hospitals in California participated in the MedImmune palivizumab trial from 1998 to 2002 [6]. It might be possible that these six hospitals, which were using palivizumab in HS-CHD patients before 2003, could affect the comparison between the Prepalivizumab Era (20002002) and the Palivizumab Era (2004-2006). In sensitivity analysis, we excluded RSV hospitalizations in these six hospitals from our study sample and found similar results. Furthermore, there might be off-label use of Synagis in HS-CHD patients prior to the 2003 recommendation, however, there are no data to assess the extent of off-label use before 2003 .

Despite these limitations, our study shows that, using actual California hospitalization data, after the 2003 AAP bronchiolistis policy revision/expansion, a decrease of only seven RSV hospitalizations per year was achieved for children with HS-CHD. The findings of our study warrant further investigation of patient compliance and costeffectiveness of RSV prophylaxis with palivizumab in patients with HS-CHD.

Open Access This article is distributed under the terms of the Creative Commons Attribution Noncommercial License which permits any noncommercial use, distribution, and reproduction in any medium, provided the original author(s) and source are credited.

\section{References}

1. American Academy of Pediatrics Committee on Infectious Diseases and Committee of Fetus and Newborn (1998) Prevention of respiratory syncytial virus infections: indications for the use of 
palivizumab and update on the use of RSV-IGIV. Pediatrics 102:1211-1216

2. American Academy of Pediatrics Committee on Infectious Diseases and Committee on Fetus and Newborn (2003) Revised indications for the use of palivizumab and respiratory syncytial virus immune globulin intravenous for the prevention of respiratory syncytial virus infections. Pediatrics 112:1442-1446

3. Booth CM, Mackillop WJ (2008) Translating new medical therapies into societal benefit: the role of population-based outcome studies. JAMA 300:2177-2179

4. Cohen SA, Zanni R, Cohen A, Harrington M, Vanveldhuisen P, Boron ML (2008) Palivizumab use in subjects with congenital heart disease: results from the 2000-2004 Palivizumab Outcomes Registry. Pediatr Cardiol 29:382-387

5. Elhassan NO, Sorbero ME, Hall CB, Stevens TP, Dick AW (2006) Cost-effectiveness analysis of palivizumab in premature infants without chronic lung disease. Arch Pediatr Adolesc Med 160:1070-1076

6. Feltes TF, Cabalka AK, Meissner HC, Piazza FM, Carlin DA, Top FH Jr, Connor EM, Sondheimer HM (2003) Palivizumab prophylaxis reduces hospitalization due to respiratory syncytial virus in young children with hemodynamically significant congenital heart disease. J Pediatr 143:532-540

7. Hall JL, Katz BZ (2005) Cost of influenza hospitalization at a tertiary care children's hospital and its impact on the cost-benefit analysis of the recommendation for universal influenza immunization in children age 6 to 23 months. J Pediatr 147:807-811

8. Joffe S, Ray GT, Escobar GJ, Black SB, Lieu TA (1999) Costeffectiveness of respiratory syncytial virus prophylaxis among preterm infants. Pediatrics 104:419-427

9. Kamal-Bahl S, Doshi J, Campbell J (2002) Economic analyses of respiratory syncytial virus immunoprophylaxis in high-risk infants: a systematic review. Arch Pediatr Adolesc Med 156:1034-1041

10. Meberg A, Bruu AL (2006) Respiratory syncytial virus infections in congenital heart defects-hospitalizations and costs. Acta Paediatr 95:404-406
11. Nuijten MJ, Wittenberg W, Lebmeier M (2007) Cost effectiveness of palivizumab for respiratory syncytial virus prophylaxis in high-risk children: a UK analysis. Pharmacoeconomics 25:55-71

12. Saez-Llorens X, Castano E, Null D, Steichen J, Sanchez PJ, Ramilo O, Top FH Jr, Connor E (1998) Safety and pharmacokinetics of an intramuscular humanized monoclonal antibody to respiratory syncytial virus in premature infants and infants with bronchopulmonary dysplasia. The MEDI-493 Study Group. Pediatr Infect Dis J 17:787-791

13. Shay DK, Holman RC, Newman RD, Liu LL, Stout JW, Anderson LJ (1999) Bronchiolitis-associated hospitalizations among US children, 1980-1996. JAMA 282:1440-1446

14. Simoes EA, Sondheimer HM, Top FH Jr, Meissner HC, Welliver RC, Kramer AA, Groothuis JR (1998) Respiratory syncytial virus immune globulin for prophylaxis against respiratory syncytial virus disease in infants and children with congenital heart disease. The Cardiac Study Group. J Pediatr 133:492-499

15. The IMpact-RSV Study Group (1998) Palivizumab, a humanized respiratory syncytial virus monoclonal antibody, reduces hospitalization from respiratory syncytial virus infection in high-risk infants. Pediatrics 102:531-537

16. The PREVENT Study Group (1997) Reduction of respiratory syncytial virus hospitalization among premature infants and infants with bronchopulmonary dysplasia using respiratory syncytial virus immune globulin prophylaxis. Pediatrics 99:93-99

17. Wegner S, Vann JJ, Liu G, Byrns P, Cypra C, Campbell W, Stiles A (2004) Direct cost analyses of palivizumab treatment in a cohort of at-risk children: evidence from the North Carolina Medicaid Program. Pediatrics 114:1612-1619

18. Yount LE, Mahle WT (2004) Economic analysis of palivizumab in infants with congenital heart disease. Pediatrics 114:16061611 